\title{
Disorder Effects in Topological StatesBrief Review of the Recent Developments
}

\author{
Binglan Wu, ${ }^{1}$ Juntao Song, ${ }^{2}$ Jiaojiao Zhou, ${ }^{1}$ and Hua Jiang ${ }^{1}$, , \\ ${ }^{1}$ College of Physics, Optoelectronics and Energy, Soochow University, Suzhou 215006, China \\ ${ }^{2}$ Department of Physics, Hebei Normal University, Hebei 050024, China
}

(Dated: November 30, 2017)

\begin{abstract}
Disorder inevitably exists in realistic samples, manifesting itself in various exotic properties for the topological states. In this paper, we summarize and briefly review work completed over the last few years, including our own, regarding recent developments in several topics about disorder effects in topological states. For weak disorder, the robustness of topological states is demonstrated, especially for both quantum spin Hall states with $Z_{2}=1$ and size induced nontrivial topological insulators with $Z_{2}=0$. For moderate disorder, by increasing the randomness of both the impurity distribution and the impurity induced potential, the topological insulator states can be created from normal metallic or insulating states. These phenomena and their mechanisms are summarized. For strong disorder, the disorder causes a metal-insulator transition. Due to their topological nature, the phase diagrams are much richer in topological state systems. Finally, the trends in these areas of disorder research are discussed.
\end{abstract}

PACS numbers: 73.61.-r, 71.23.-b, 73.43.-f

\section{INTRODUCTION}

Topological states, including gapped topological insulators and gapless topological semimetals, have become a focus of the condensed matter research 1 [ 8 . In condensed matter systems, the first well-known insulating topological state is quantum Hall effect (QHE) under a strong magnetic field ${ }^{9}$. Subsequently, the quantum anomalous Hall effect (QAHE), a topological state similar as the QHE but without magnetic field, was proposed in $1988^{10}$ and observed in $2013^{11}$. In 2005, Kane et al made a great step in the topological states research. They proposed the two-dimensional $Z_{2}$ topological insulator - quantum spin Hall effect (QSHE) in graphene, which extends the topological state into the class of systems protected by time reversal symmetry 12113 . Soon afterwards, the concept of symmetry protected topological states is broaden into three-dimension ${ }^{14 / 15}$ and other discrete symmetries ${ }^{7 / 16}$. Besides the insulating systems, the topological states can also exist in gapless systems ${ }^{17 / 18}$. More recently, topological semimetals, containing both Weyl semimetals and Dirac semimetals, were experimentally verified ${ }^{1920}$, soon after they were predicted in the corresponding materials2122. The topological states are different from normal metallic and insulating states because of the existence of nontrivial topological order, which originates from the global properties of all electrons below the Fermi energy ${ }^{23}$ and can be characterized by various types of topological invariant numbers 2113223 . Due to the nontrivial topological order, corresponding gapless states emerge on the surface, leading to numerous exotic properties in topological systems. These properties have been reviewed in references $\frac{1}{1} 8$.

Experimentally, disorder is ubiquitous because of the defects in manufacturing processes and usually plays a dominant role in the transport properties of the samples being studied. Due to their unique electric structures, the response of topological states to disorder is fundamentally different from that in normal metals and insulators 1334923133 . Physically, the topological invariant number can take on only a few discrete values. Therefore, the topological order cannot be easily disrupted by weak perturbations. That is to say, quantized transport that is robust against weak disorder can be obtained in various of topological systems. Moreover, arising from their topological nature, topological states also show unconventional properties under moderate and strong disorder. Thus, studies of disorder effects can not only give a comprehensive understanding about topological states, but also offer a route map for the application of 
topological states. In this paper, based on several of our finished works in the last few years 30 , w3 three topics covered by recent studies of disorder effects in topological states. We note that there are other topics

related to disorder, such as the weak anti-localization $26 \mid 27$, the chiral anomaly effects $28 \mid 29$ etc, which are not reviewed here.

The rest of this paper is organized as follows. In Section 2, the robustness of topological states against weak disorder is reviewed. In section 3, we present how various type of disorder create topological insulator states from normal insulating or metallic states. In section 4, the main results from studies of the metal-insulator transition in topological states system are summarized. Finally, a brief conclusion and an outlook for future disorder research are given in Section 5 .

\section{ROBUSTNESS OF TOPOLOGICAL STATES}

Searching for material states with low-power dissipation is one of the greatest challenges in modern fundamental physics and materials science. In a real experimental sample, disorder always exists to some degree. A carrier propagating inside the sample will inevitably collide with disorder sites and be scattered. However, whether such a collision can cause energy dissipation depends on the ability of the scattering to induce backscattering processes - carriers propagating in the forward channel are scattered into the backward channel. In a normal metal, due to the spatial overlap between the forward and backward channels, backscattering can occur even for weak disorder. Therefore, the resistance (conductance) in a normal metal is not universal but in general increases (decreases) with the disorder strength. Transport in such a system is dissipative. In sharp contrast, in various kinds of topological systems, the spatial separation between the forward and backward channels and the existence of a special discrete symmetry (e.g. time-reversal symmetry) forbid backscattering 34 . As a result, carriers can propagate without dissipation. Thus, the resistance (conductance) in these systems is quantized and is insensitive to the presence of weak disorder. Such exotic phenomenon, namely the robustness of topological states, makes their host materials ideal platforms for testing fundamental physics principles and achieving low-power dissipation in electronic/spintronic devices.

That topological states are robust against weak disorder was first discovered in a milestone experiment on quantum Hall effects 9 . Klitzing et al observed quantized Hall resistance and zero longitudinal resistance, independent of the material details. Subsequently, such phenomena have been extensively studied both within the context of quantum Hall effect and in various other topological states 35 . In the following, as examples, we demonstrate the robustness of the topological states and the mechanisms of this behavior in two recently discovered systems: (i) quantum spin Hall effects (QSHE) in HgTe/CdTe quantum wells 30 and (ii) new proposed $Z_{2}=0$ topological insulators with emerging robust helical surface states 31 . 


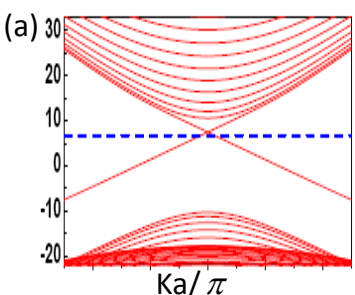

(b)

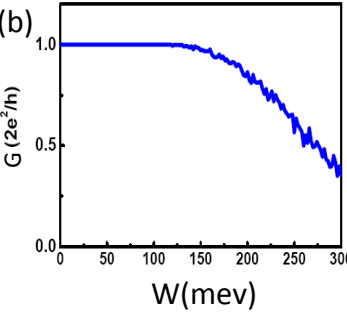

(c)
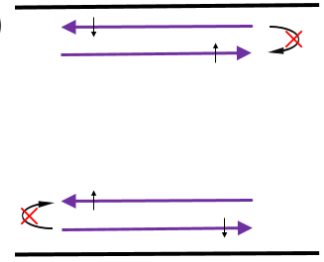

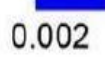

0.02 $0.2\left(e^{2} \mathrm{~V} / \mathrm{h}\right)$

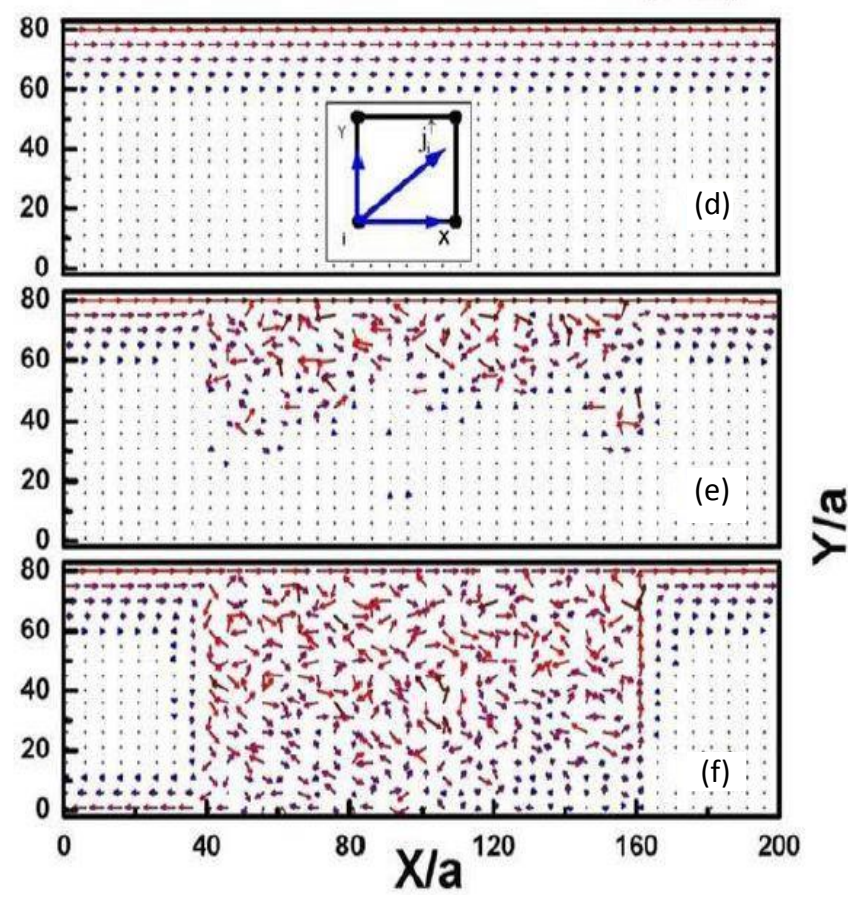

Fig. 1. (color online) (a) Typical energy spectrum for a HgTe/CdTe quantum well strip in the QSHE region. (b) The conductance $G$ vs disorder strength $W$ in a two terminal device. The Fermi energy is located inside the inverted gap. (c) Schematic of helical edge states propagation in the boundary of sample. On a given edge, the carriers with opposite spin polarizations propagate in opposite directions. (d) -(f) Configurations of the local current flow vector in a device with central region size $L_{x}=200 a, L_{y}=80 a$ under disorder strength (d) $W=0,(\mathrm{e}) W=110 \mathrm{meV}$, and (f) $W=220 \mathrm{meV}$. The inset of (d) is the schematic of local current flow vector. The direction and length of the arrows represent the local current direction and magnitude. The order of magnitude of the local currents are displayed in the color bar. Adapted from Ref.

For $\mathrm{HgTe} / \mathrm{CdTe}$ quantum wells of moderate thickness, due to the strong spin-orbit coupling in this material, the system can enter a new topological phases-the QSH phase ${ }^{24 \mid 42[43}$. In Fig. 1(a), the energy spectrum for the quasi one-dimensional strip is plotted. There exists an inverted bulk energy gap with two degenerate energy bands (helical edge states) that cross inside the gap. When the Fermi energy is located inside the gap, e.g. $\varepsilon_{F}=7 \mathrm{meV}$, the QSH phase is established. To show how the transport properties of QSH phase are influenced by the disorder, a device with two terminals and a disordered central region is considered. Fig. 1(b)shows the two terminal conductance $G$ versus the disorder strength $W$ for the state shown in Fig. 1(a). For a range of disorder strength $W \in[0 \mathrm{meV}, 150 \mathrm{meV}]$, the conductance $\mathrm{G}$ takes the quantized value $2 e^{2} / h$. Such an observation means that the QSHE is robust against weak disorder. Preliminarily, the behavior of the conductance $G$ can be understood on the basis of spatial distribution of the helical edge states, as shown in Fig. 1(c). In the absence of disorder, the spin up channel on the top boundary and the spin down channel on bottom boundary give rise to the $G=2 e^{2} / h$. In the presence of nonmagnetic disorder, timereversal symmetry forbids the backscattering on a given edge ${ }^{34}$. Since the backscattering between the edge channels on the opposite boundaries decays exponentially due to the spatial separation, $G$ shows a plateau with $G=2 e^{2} / h$ without fluctuations.

Next,in order to illustrate clearly the evolution of the conductance $G$ versus disorder, the typical distributions of local currents in the device are plotted in Fig. 1(d)-(f). Note that only the local currents for spin-up subsystem are demonstrated. The local currents for the spin-down subsystem can be directly obtained by applying the time-reversal symmetry. In a clean sample[Fig. 1(d)], the local currents are mainly located on the upper edge and their values 
decay exponentially toward the bulk. The local currents spread into the bulk and the edge channel is broadened when disorder is introduced [Fig. 1(e)]. However, not until the disorder strength $W$ exceeds the critical value $W_{c}$, can the spreading local current connect with the bottom edge channels, which have the opposite chirality. At this point, effective backscattering [Fig. 1(f)] can occur, leading to a decrease in the conductance $G$ between the two terminals. These pictures explain why $G$ is robust against weak disorder and how it is destroyed in the strong disorder limit.
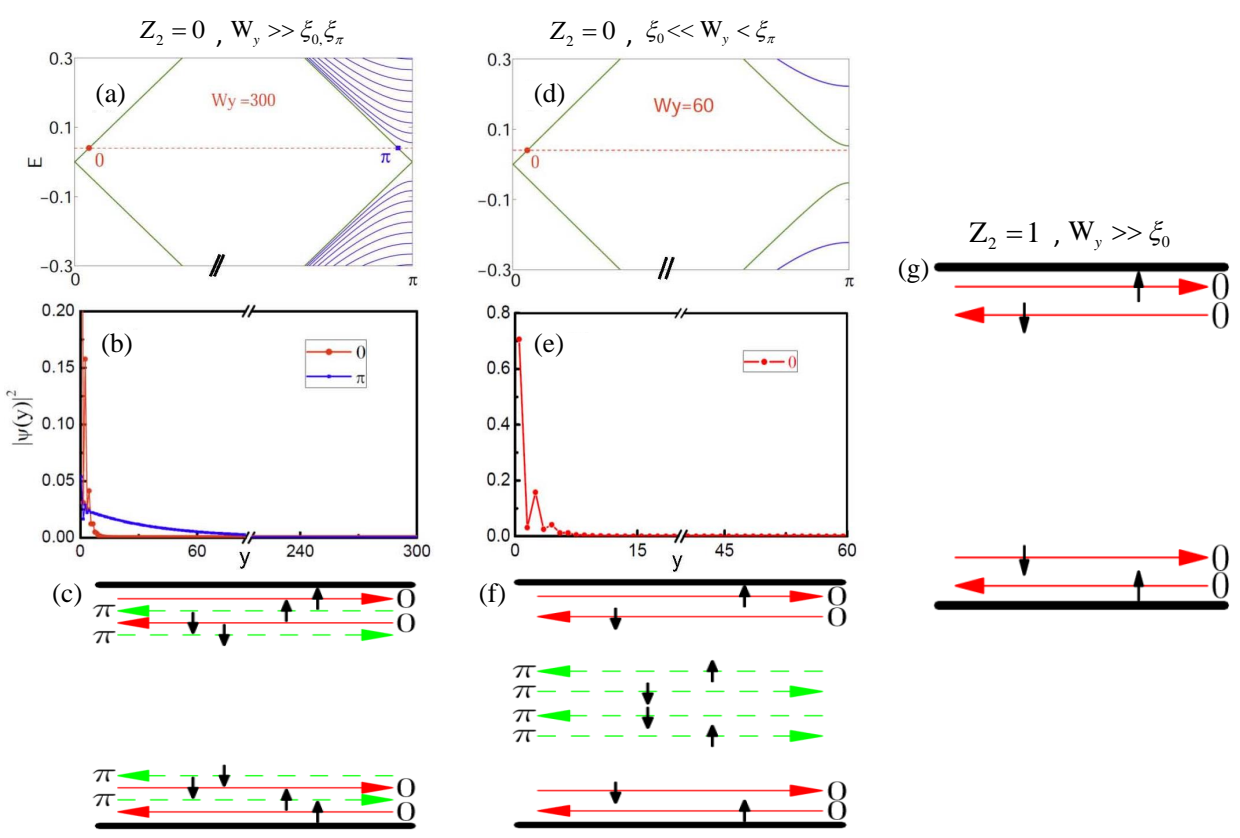

Fig. 2. (color online)(a),(d) One dimensional energy bands for the $2 \mathrm{D}$ anisotropic BHZ stripe with $Z_{2}=0$ and sample width $\mathrm{W}_{\mathrm{y}}=300$ (a), 60 (d) described in the main text . (b),(e) show distribution of edge states in real space corresponding to (a) and (d) with fixed Fermi energy.(c) and (f) show schematic plots of helical edge modes corresponding to (a) and (d), respectively. The vertical arrows represent the electron spin. In panel (f), the helical edge channels around $k_{x}=\pi$ are hybridized due to finite size confinement, leaving one pair of helical edge channels around $k_{x}=0$. Thus, these conducting channels resemble the helical edge channels in a $Z_{2}=1$ QSHE. Panels (c),(f) and (g) are adapted from Ref $\frac{31}{\text {. }}$

Topological insulators protected by time-reversal symmetry are characterized by the topological invariant number $Z_{2}$. There exist odd pairs of helical surface/edge states in topological insulators with $Z_{2}=1$, which are robust and insensitive to material details and to external perturbations, as demonstrated in Fig. 1. However, most real materials with time reversal symmetry have $Z_{2}=0$ topological order, and systems with $Z_{2}=1$ topological order are very rare. Indeed, the QSHE (two-dimensional topological insulator with $Z_{2}=1$ ) has been experimentally confirmed only in $\mathrm{HgTe} / \mathrm{CdTe}^{24}$ and InAs/GaSb ${ }^{\underline{44 \mid 45}}$ quantum wells. It is natural to ask, whether the $Z_{2}=1$ is a necessary requirement to realize topological surface/edge states.

According to the popular view, $Z_{2}=0$ topological systems have even pairs of helical surface/edge states, which are fragile in the presence of disorder $40 \mid 46$. However, we find that for some $Z_{2}=0$ systems, robust transport can be engineered using a combination of finite size confinement and anisotropy 31 . With the help of the anistropic Bernevig-Hughes-Zhang (BHZ) mode $\stackrel{42[43}{4}$, we demonstrate how robustness against disorder arises in such topological states.

In an anisotropic, $Z_{2}=0 \mathrm{BHZ}$ stripe, for certain parameters, there are two pairs of helical edge states around $k=0$ and $k=\pi$ [Fig. 2(a)]. The 0 helical edge states are located close to the edge, while the $\pi$ helical edge states are more delocalized toward the bulk [Fig. 2(b)]. When the stripe is sufficiently wide, [i.e. $W_{y}=300$, which is much greater than the decay length of both the 0 and $\pi$ helical edge channels], both $k=0$ and $\pi$ helical edge bands are 
nearly gapless as expected. As a consequence, the Fermi energy crosses the $k=0$ helical edge states and counterpropagating $\pi$ edge states, which also have substantial real-space overlap[Fig.2(c)]. The disorder can heavily couple these two states and cause strong backscattering between the $k=0$ and $k=\pi$ edge channels, leading to complete localization. In transport experiments, such a stripe then resembles a normal insulator. In contrast, when the stripe becomes narrow [i.e. $W_{y}=60$ ], the 0 helical edge bands remain almost gapless, while the coupling of extended $\pi$ helical edge states opens a remarkable hybridization gap $\Delta$ [Fig. 2(d)]. When the Fermi energy is located inside the gap $\Delta$, only the $k=0$ helical edge states exist. Therefore, the $k=0$ helical edge states are well separated with the $k=\pi$ states. In addition, since the decay length of 0 helical edge states, $\xi_{0}$, is much shorter than the sample width $W_{y}$, the $k=0$ helical edge states on the top edge are also well separated with that on the bottom edge [Fig. 2(e) and (f)]. From a transport point of view, the conducting edge channels in Fig.2(f) $\left[Z_{2}=0\right]$ are similar to those in Fig. 2(g) and Fig. 1(c) $\left[Z_{2}=1\right]$. Conclusively, then despite having a topological number $Z_{2}=0$, the helical edge states in Fig. 2(f) emerge as being robust.

In order to quantitatively assess the robustness of these emerging $Z_{2}=0$ helical edge states, their transport properties under nonmagnetic disorder in a two terminal device and a $\pi$-bar device [Fig. 3(a) and 3(e)] are simulated. The results are demonstrated in Fig. 3(c) and 3(e), respectively. For comparison, the transport properties of these two devices incorporating (i) normal $Z_{2}=0$ states with two pairs of helical edge states [Fig. 3(b) and 3(f)] and (ii) $Z_{2}=1$ QSHE [Fig. 3(d) and 3(h)] are also obtained. In the case of emergent helical edge states, the two terminal conductance $G_{12,12}$ shows a quantized plateau $2 e^{2} / h$ in the clean limit. When nonmagnetic disorder is introduced, the $2 e^{2} / h$ plateau remains unchanged without fluctuation [Fig. 3(c)]. Meanwhile, the nonlocal conductance $G_{14,23}$ in the $\pi$-bar device shows well quantized plateaus at $4 e^{2} / h$, irrespective of details of the terminals and the strength of the strong disorder [Fig. 3(g)]. Therefore, the transport properties of the emergent helical states are completely different from normal $Z_{2}=0$ states cases, where $G_{12,12}$ and $G_{14,23}$ are found that are found that are fragile against disorder. Instead, the $G_{12,12}$ and $G_{14,23}$ cases behave exactly like a $Z_{2}=1$ QSHE system, see Figs. 3(d) and 3(h), and the experimental results in $\underline{45}$. The two-terminal perfect $2 e^{2} / h$ plateau and the $\pi$-bar perfect $4 e^{2} / h$ plateau plausibly provide a transport definition of robust helical edge states in these $Z_{2}=0$ systems. In a $Z_{2}=1$ QSHE, the robustness of the edge conduction is derived from its intrinsic topological character. On the other hand, the robust helical edge states in the $Z_{2}=0$ model arise from the fact that the backscattering is detuned by the finite size confinement.

Besides the anisotropic BHZ model, the $Z_{2}=0$ topological systems involving helical surface states with emergent robustness have been extended into several $2 \mathrm{D}$ and $3 \mathrm{D}$ models 314749 . In addition, two systems built on realistic materials: (i) Dirac semi-metal with appropriate width and thickness confinement $\frac{50}{}$ and (ii) a bismuth (111) film with a thickness of between 20 and $70 \mathrm{~nm} 3151$ are proposed to host robust helical surface states. Recent relevant experiments have shown the clues of the existence of such topological states in the latter proposed material system 15152 . It is worth noting that the proposed $Z_{2}=0$ systems have additional exotic properties not present in $Z_{2}=1$ QSHE, which can be utilized to fabricate new topological devices 50 . 

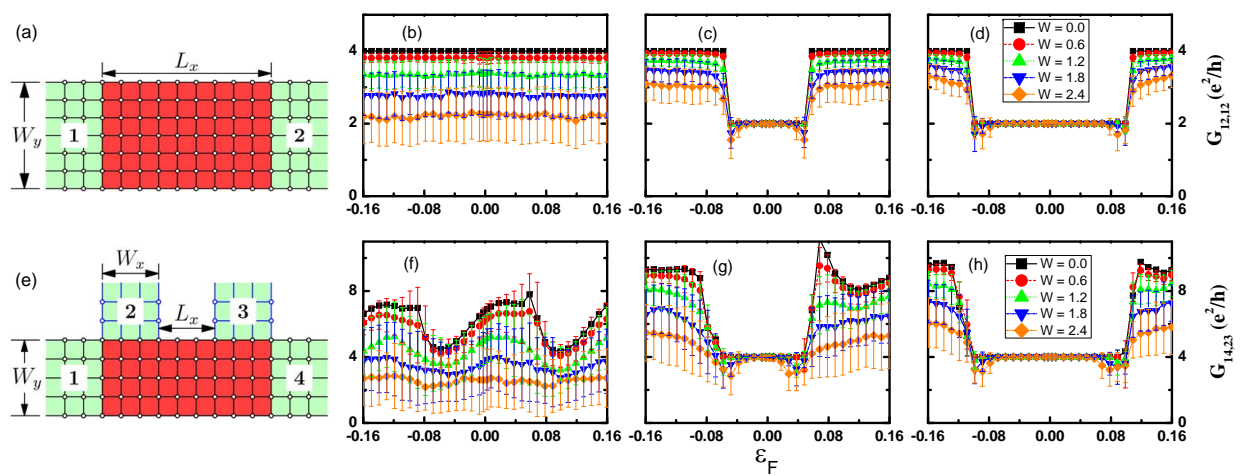

Fig. 3. (color online)(a),(e) Illustration of two-terminal and $\pi$-bar devices . The Anderson disorder exists only in the central (red) region. The size parameters are $L_{x}=120, \mathrm{~W}_{\mathrm{x}}=120, \mathrm{~W}_{\mathrm{y}}=60$. (b)C(d) Two-terminal conductance $G_{12,12}$ of device (a); (f)C(h) nonlocal conductance $G_{14,23}$ of device (e) vs the Fermi energy $\varepsilon_{F}$ for different disorder strengths $W$ for the cases of a normal $Z_{2}=0$ system (b) and (f); $Z_{2}=0$ topological system with emergent robust helical edge states (c) and (g) and $Z_{2}=1$ QSHE (d) and (h). $G_{14,23}$ means that the current $I_{14}$ is injected from terminal 1 to 4 and the voltage $V_{23}$ is measured between terminal 2 and 3 and $G_{14,23}=\frac{V_{23}}{I_{14}}$. The error bars denote the conductance fluctuation. Adapted from ref 31

\section{DISORDER INDUCED TOPOLOGICAL INSULATORS}

Besides destroying topological states, in special systems, moderate disorder can also produce topological states. The first well studied disorder induced topological state was the topological Anderson insulator (TAI). Specifically, in the clean limit, the system is in an ordinary insulator or metal. With the introduction of disorder, it enters into a topological insulator state with robust transport. This TAI was predicted to arise in $\mathrm{HgTe} / \mathrm{CdTe}$ quantum wells by Shen's group ${ }^{53}$ and our group 30 . This type of TAI was then extended to many other systems $54 \sqrt[65]{6}$.

Let us begin with a review of how TAI arises in $\mathrm{HgTe} / \mathrm{CdTe}$ quantum wells. In the clean limit, the system is described by the BHZ modei

$$
\begin{aligned}
\mathcal{H}_{l}(\vec{k}) & =\left(\begin{array}{cc}
h_{0}(\vec{k}) & 0 \\
0 & h_{0}^{*}(-\vec{k})
\end{array}\right), \\
h_{0}(\vec{k}) & =\varepsilon(\vec{k})+d_{\alpha}(\vec{k}) \sigma_{\alpha},
\end{aligned}
$$

where $\sigma_{\alpha}[\alpha=x, y, z]$ are the Pauli matrices, and

$$
\begin{aligned}
\varepsilon(\vec{k}) & =C-D\left(k_{x}^{2}+k_{y}^{2}\right), \\
\mathbf{d}(\vec{k}) & =\left[A k_{x}, A k_{y}, M-B\left(k_{x}^{2}+k_{y}^{2}\right)\right] .
\end{aligned}
$$

$A, B, C, D, M$ are material parameters. Specially, $M$ is the topological mass, which can be tuned continuously through the thickness of the HgTe layer. If $M<0$, the bulk band is inverted and the system becomes to a QSHE [Fig. 4(b)]. In contrast, if $M>0$, the system becomes a normal insulator [Fig. 4(e)].

In Fig. 4(a) and 4(d), which is taken from Ref ${ }^{53} \mathrm{Li}$ et al plot the two terminal conductance $G$ versus the disorder strength $W$ for different Fermi energies $\varepsilon_{F}$. When the Fermi energy $\varepsilon_{F}$ is in the valence band, then for both $M<0$ and $M>0, G$ quickly decreases to zero as the disorder strength $W$ increases [green line in 4(a) and 4(d)]. In sharp contrast, if $\varepsilon_{F}$ is located near the edge of the conduction band, for both $M<0$ and $M>0 G$ first decreases when Anderson disorder is introduced. Intriguingly, continuously increasing the disorder strength $W$ does not lead to the localization of the system, but makes $G$ increase again up to a quantized value. This value is maintained for a certain 
range without fluctuations [blue line in $4(\mathrm{a})$ and $4(\mathrm{~d})]$. As described in section II, the $2 e^{2} / h$ plateau signals that the system has entered a new topological state, referred above as the TAI. Further, the nature of the TAI is clarified by the phase diagrams shown in Fig. 4(c) $[M<0]$ and in Fig. 4(f) $[M>0]$. Significantly, for $M>0$, there are no topological helical edge states in the clean limit [Fig. 4(e)]. The quantized plateau cannot be attributed to the coexistence of the bulk and edge states, and the disorder causes the localization of the bulk states. It therefore seems that Anderson disorder creates the helical edge state, and leads to robust transport.

(a)
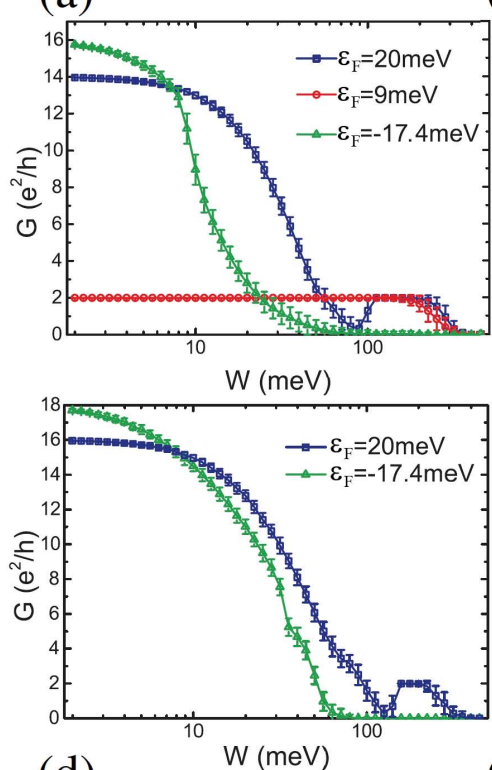

(d) (b)

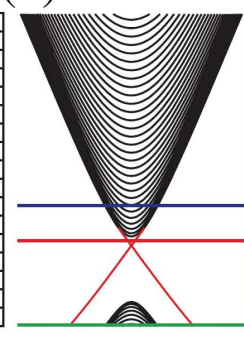

(c)
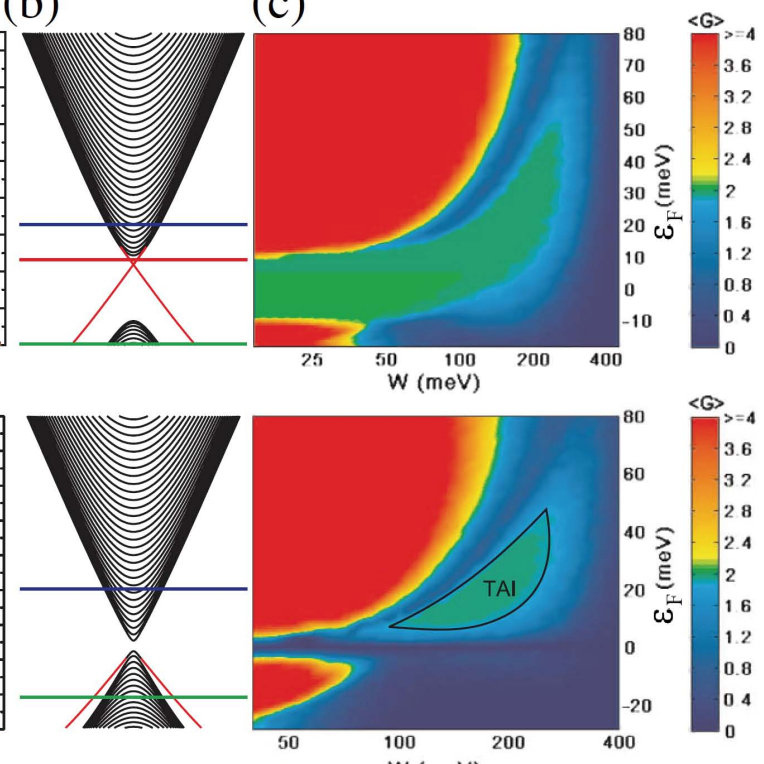

(e)

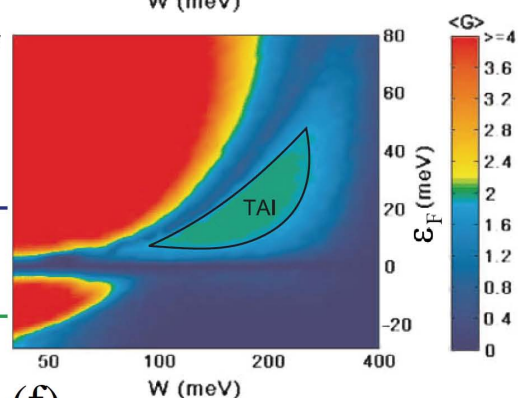

(f)

Fig. 4. (color online) Conductance of disordered strips of HgTe/CdTe quantum wells. (a)-(c) and (d)-(f) show results for an inverted quantum well with $M=-10 \mathrm{meV}$ and for a normal quantum well with $M=1 \mathrm{meV}$, respectively. (a) The conductance $G$ versus disorder strength $W$ at three values of Fermi energy. The error bars represent the conductance fluctuations. (b) One dimensional energy spectrum. The vertical scale (energy) is the same as in (c) and the horizontal lines correspond to the values of the Fermi energy considered in (a). (c) Phase diagram showing the conductance $G$ as a function of both disorder strength $W$ and Fermi energy $\varepsilon_{F}$. The panels (d), (e), and (f) are the same as (a),(b), and (c), but for $M>0$. The TAI phase is shown in green region. In all panels, the strip width is $500 \mathrm{~nm}$ and the length is $5000 \mathrm{~nm}$ in (a) and (d), and $2000 \mathrm{~nm}$ in (c) and (f ). Adapted from Ref.553.

To validate this assumption, we study the evolution of the local current configurations under different disorder strengths [Fig. 5] $]^{30}$. The parameters were set to be $M=2 \mathrm{meV}$ and $\varepsilon_{F}=18 \mathrm{meV}$ [Fig. (5a)]. The plot of $G$ vs $W$ in Fig. 5(b) can be subdivided into four regions (i) without disorder, (ii) before the anomalous plateau, (iii) on the anomalous plateau, and (iv) after the anomalous plateau. Typical spatial distributions of the local currents are plotted in Fig. 5(h)-(k), respectively. The most interesting phenomena are to be found in region (iii) [Fig. 5(i)]. Here, the local currents in the bulk decline to zero while the residual currents flow around the upper edge with little scattering [only the spin up subsystem is considered]. In addition, throughout region (iii), the bulk transport vanishes and the edge transport shows the same behavior as in the traditional QSHE region [Fig. 1(d)-(f)]. The local currents shown in Fig. 5 provides strong evidence that disorder leads to the helical edge states and hence to the TAI.

Based on the discoveries described in references ${ }^{30 \mid 53}$, Groth et al. present an effective medium theory that explains the physical origin of the TAl ${ }^{66}$. Disorder can induce a self-energy $\Sigma$. In the self-consistent Born approximation (SCBA), $\Sigma$ is given by

$$
\Sigma=\frac{W^{2}}{12}(a / 2 \pi)^{2} \int_{\mathrm{BZ}} d \vec{k}\left[\sigma_{\alpha}\left(\varepsilon_{F}^{+}-h_{0}(\vec{k})-\Sigma\right)^{-1} \sigma_{\alpha}\right]
$$


Here $\sigma_{\alpha}$ denotes the type of disorder, and $a$ is the lattice constant. $\Sigma$ can be decomposed into the form $\Sigma=$ $\Sigma_{0} \sigma_{0}+\Sigma_{x} \sigma_{x}+\Sigma_{y} \sigma_{y}+\Sigma_{z} \sigma_{z}$. Therefore, the self energy, $\Sigma$, makes a correction to the original Hamiltonian $h_{0}(\vec{k})$ and renormalizes the topological mass and the Fermi energy:

$$
\bar{M}=M+\operatorname{Re} \Sigma_{z}, \quad \overline{\varepsilon_{F}}=\varepsilon_{F}+\operatorname{Re} \Sigma_{0} .
$$

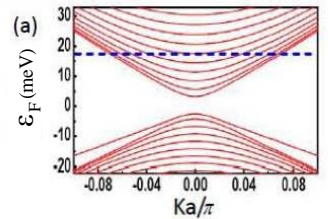

(c)

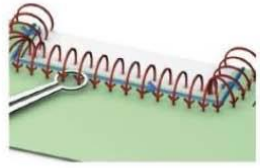

(e)

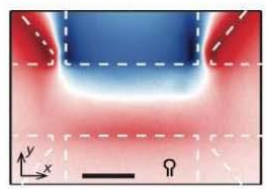

Conduction band

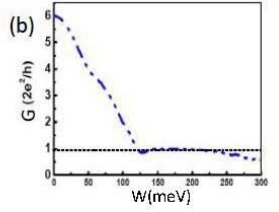

(d)

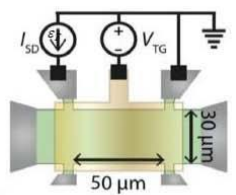

(f)

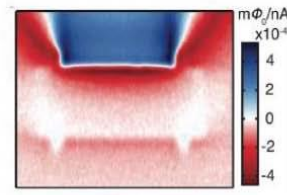

Bulk gap

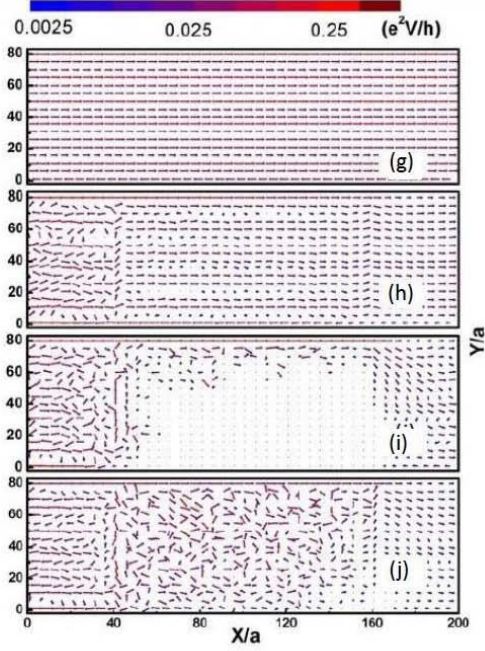

$\frac{\pi}{>}$

Fig. 5. (color online ) (a) Typical one-dimensional energy spectrum for a normal $\mathrm{HgTe} / \mathrm{CdTe}$ quantum well strip. (b) Conductance $G$ vs disorder strength $W$. (g)-(j) Configurations of the local current flow vector for the strip with the same sample sizes as for Fig. 1, and with the disorder strength set at (g) $W=0$, (h) $W=100 \mathrm{meV}$, (i) $W=150 \mathrm{meV}$, and (j) $W=250 \mathrm{meV}$. In (a),(b),(g)-(j), The Fermi energy and topological mass are set to be $\varepsilon_{F}=18 \mathrm{meV}$ and $M=2 \mathrm{meV}$. (c) Sketch of the configurations for the local current flow measurement. The magnetic field (red) generated by the current (blue) is measured by detecting the flux through the SQUID's pickup loop. (d) Schematic of a Hall bar. (e)(f) Typical measured distributions of the local current when Fermi energy is inside the conduction band (e) and the bulk gap (f), respectively. (a)(b)(g)-(j) are reproduced from Ref ${ }^{30}$. (c)-(f) are adapted from Ref 108 .

Groth et a ${ }^{\sqrt{66}}$ found that the Anderson type of disorder $\left[\sigma_{0, z}\right]$ can make a negative correction $\left[\operatorname{Re} \Sigma_{z}<0\right]$ to the topological mass $M$. Thus, it can lead a transition of $\bar{M}$ from positive to negative by increasing the disorder strength. Moreover, due to lacking the particle-hole symmetry of $h_{0}(\vec{k})$, the Anderson disorder also makes a negative correction to the Fermi energy $\varepsilon_{F}$. When $\varepsilon_{F}$ is in the conduction band, for both $M>0$ and $M<0$, the Anderson disorder can tune the renormalized Fermi energy $\overline{\varepsilon_{F}}$ into the inverted gap $[-|\bar{M}|,|\bar{M}|]$. The TAI phase is then established. In contrast, when $\varepsilon_{F}$ is in the valence band, $\overline{\varepsilon_{F}}$ is shifted away from the gap. $G$ quickly decays to zero and no quantized conductance appears.

If the effective medium theory is correct, one can deduce from Eq.(3) that the emergence of TAI is highly relevant to the type of disorder. Concretely, the $\sigma_{z}$ term exists in $\left(\varepsilon_{F}^{+}-h_{0}(\vec{k})-\Sigma\right)^{-1}$. While Anderson disorder $\left(\sigma_{0, z}\right)$ makes a negative correction $\left(\sigma_{0} \sigma_{z} \sigma_{0}=\sigma_{z} \sigma_{z} \sigma_{z}=\sigma_{z}\right)$ to the topological mass $M$, the bond disorder $\left(\sigma_{x, y}\right)$ results in a positive correction $\left(\sigma_{x} \sigma_{z} \sigma_{x}=\sigma_{y} \sigma_{z} \sigma_{y}=-\sigma_{z}\right)$ to $M$. To see this more clearly, in Fig. 6, we compare the conductance phase diagrams for Anderson disorder and bond disorder in the $\mathrm{HgTe} / \mathrm{CdTe}$ quantum wells with $M=1 \mathrm{meV} \sqrt{68}$. It can be seen from Fig. 6(a), that at moderate Anderson disorder and with an appropriate Fermi energy, a clear TAI phase [green region] is present, and such a TAI phase should correspond to a negative renormalized topological mass $\bar{M}$ [blue region in Fig. 6(c)]. When referring to the bond disorder case, for any disorder strength $W$ and Fermi energy $\varepsilon_{F}, \bar{M}$ is always positive. Correspondingly, there is no indication of the TAI phenomenon in Fig. 6(b). From Fig. 6, one can conclude that normal Anderson disorder gives rise to the TAI phenomenon and bond disorder destroys it. 
The results not only verify the validity of the effective medium theory, but also are important for the experimental realization of the TAI phase.

To data, the physical properties of the TAI phase have been extensively studied $[\sqrt[69]{75}$, and several significant advances are worth noting. In Fig. 4(f) and Fig. 6(a), since it is surrounded by a normal Anderson insulator phase, the TAI phase was initially considered to be a new topological phase. Later, using a three dimensional phase diagram [disorder strength W, Fermi energy $\varepsilon_{F}$ and topological mass $\left.M\right]$, Prodan found that the TAI pahse is not a distinct phase but part of the quantum spin-Hall phase, because these two phases can be adiabatically connected by varying the topological mass 69 . Xu et al studied the phenomenon in a very large sample, and a complete phase diagram of the TAI phase was obtained 70 . They found that the TAI phase region was much lager than what SCBA theory predicted. The reason is that the TAI can exist in a mobility gap rather than an inverted band gap 70171 .

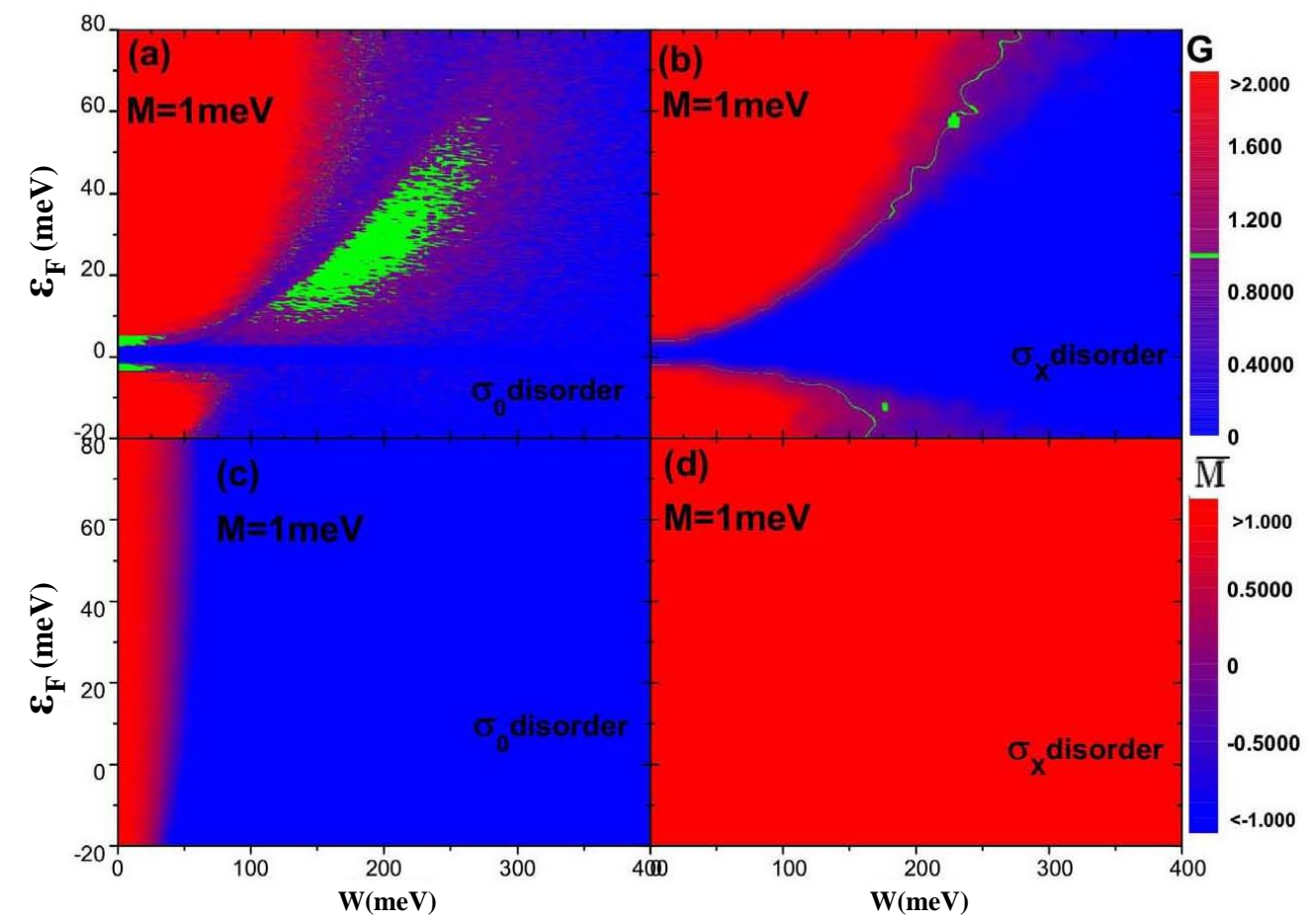

Fig. 6. (color online) The conductance $G$ (a), (b) and renormalized topological mass $\bar{M}$ (c), (d) vs disorder strength $W$ and Fermi energy $\varepsilon_{F}$ for the $\mathrm{HgTe} / \mathrm{CdTe}$ model with $M=1 \mathrm{meV}$. Here, $\sigma_{0}$ and $\sigma_{x}$ correspond to Anderson disorder and bond disorder, respectively. Adapted from Ref. 68

In what follows, we introduce another type of topological state induced by disorder. Compared to the above TAI state, there are several differences. First, such a state is not triggered by the disorder strength but the randomness of the adatom configuration. Second, such a state cannot be explained by the effective medium theory. Third and of the greatest importance, contrary to the common view, such disorder induced topological states are much easier to be realized than the normal insulator state in the periodic case.

Because of the outstanding properties of its electronic structure, graphene is considered as an ideal platform to host topological phases. For instance, recent first-principles calculations proposed that certain nonmagnetic adatoms (e.g., indium and thallium) could enhance the intrinsic spin-orbit coupling to give rise to the QSHE adatoms (e.g., 3d and 5d transition metals) could induce sizable Rashba spin-orbit coupling and magnetization to produce the quantized anomalous Hall effect (QAHE) 79 . Though great theoretical progress has been achieved, no experimental observation has been reported. There are two major reasons preventing the experimental exploration of 
these novel states. (i) All the calculations are based on the periodic adsorption condition, which is beyond existing experimental capabilities. (ii) The formation of topological states is highly dependent on the adatom configurations. At certain coverage rates, the system is a trivial insulator.

Let us first explain why the topological nontrivial state and trivial insulator state can exist in the periodic adsorption case with different coverage rates. As illustrated in Fig. 7(a) and (b), the tight-binding Hamiltonian of graphene with non-magnetic adatoms can be written as $32 \mid 82$ :

$$
\begin{aligned}
H & =H_{0}+H_{\mathrm{so}}+H_{\mathrm{U}} \\
& =-t \sum_{\langle i j\rangle, \alpha} c_{i \alpha}^{\dagger} c_{j \alpha}+i \lambda_{\mathrm{SO}} \sum_{\langle\langle i j\rangle\rangle \in \mathcal{R}, \alpha \beta} \nu_{i j} c_{i \alpha}^{\dagger} s_{\alpha \beta}^{z} c_{j \beta}+U \sum_{i \in \mathcal{R}, \alpha} c_{i \alpha}^{\dagger} c_{i \alpha} .
\end{aligned}
$$

The presence of the adatom at $\mathcal{R}$ not only enhances the intrinsic SOC term $\left(H_{\mathrm{so}}\right)$ but also generates the on site potential term $\left(H_{\mathrm{U}}\right)$ with respect to the surrounding carbon atoms. $H_{\mathrm{so}}$ induces a topologically nontrivial gap $\Delta_{\text {so }}$ at both $K$ and $K^{\prime}$ with magnitude

$$
\begin{aligned}
\Delta_{\mathrm{so}} & \propto \sum_{\mathcal{R}} H_{\mathrm{so}}(\mathcal{R}) e^{i(K-K) \cdot R}=\sum_{\mathcal{R}} H_{\mathrm{so}}(\mathcal{R}) e^{i\left(K^{\prime}-K^{\prime}\right) \cdot R} \\
& =\sum_{\mathcal{R}} H_{\mathrm{so}}(\mathcal{R})
\end{aligned}
$$

and $H_{\mathrm{U}}$ induces the inter-valley scattering, which results in a topological trivial gap $\Delta_{U}$ with magnitude

$$
\Delta_{U} \propto \sum_{\mathcal{R}} H_{\mathrm{U}}(\mathcal{R}) e^{i\left(K-K^{\prime}\right) \cdot \mathcal{R}}
$$

When adatoms form a $\sqrt{3} n \times \sqrt{3} n$ or $3 n \times 3 n$ supercell, a finite $\Delta_{U}$ can be obtained since the factor $e^{i\left(K-K^{\prime}\right) \cdot \mathcal{R}}=1$ for all $\mathcal{R}$. Because the on-site potential $U$ is much larger than the intrinsic SOC $\lambda_{\text {so }}$, the topological trivial gap $\Delta_{U}$ exceeds the topological nontrivial gap $\Delta_{\text {so }}$, resulting in a trivial insulator state. For other periodic adsorption cases, the inequivalent $e^{i\left(K-K^{\prime}\right) \cdot \mathcal{R}}$ for various $\mathcal{R}$ vanishes the inter-valley scattering $\left[\Delta_{U} \rightarrow 0\right]$. Since $\Delta_{\text {so }}>\Delta_{U}$, a quantum spin Hall state is realized 3 .

Interestingly, when the adatoms are nonuniformly distributed in space, the factor $e^{i\left(K-K^{\prime}\right) \cdot \mathcal{R}}$ becomes randomized. As a result, even when adatom is at the coverage rate $\frac{1}{3 n^{2}}$ or $\frac{1}{9 n^{2}}$, such randomization reduces $\Delta_{U}$ from a large value in periodical case to nearly zero in the random adsorption case. We note the renormalization process for $\Delta_{U}$ cannot be described by the effective medium case. In sharp contrast, the topologically nontrivial gap $\Delta_{\text {so }}$ is caused by zero momentum scattering. From Eq. (6), the randomization of the adatom distribution plays a negligible role in determining the magnitude of $\Delta_{\text {so }}$. Therefore, one can expect a quantum phase transition from trivial insulator state $\left[\Delta_{\mathrm{so}}<\Delta_{U}\right]$ to a topological insulator state $\left[\Delta_{\mathrm{so}}>\Delta_{U}\right]$ with the introduction of the spatial randomness of the adsorbates.

As an example, we study the possibility of realizing QSHE states in graphene with randomly distributed adsorbates. Fig. 7 (c) shows the results of a simulation of the transport properties of the system with a $11.1 \%$ coverage ratio $[3 \times 3$ supercell]. For the case of periodically distributed adatoms [Fig. 7(a)], a zero two terminal conductance $G=0$ is observed in the region where $\varepsilon_{F} \in[0.117 t, 0.124 t]$, signaling a trivial insulator. However, when the adatoms become randomly distributed [Fig. 7(b)], a quantized plateau $G=2 e^{2} / h$ with vanishing fluctuation emerges within the range $\varepsilon_{F} \in[0.116 t, 0.132 t]$, indicating that the system turns into the quantum spin Hall phase. Fig. 7(d) shows a simulation 
of the transport properties of the system with a $6.25 \%$ coverage ratio [ $4 \times 4$ supercell]. The robust quantized plateau $G=2 e^{2} / h$ for both two cases shows that the topological phase predicted from first-principles is not affected by the randomization of the adatom configurations. In Fig. 7(e), we show that randomization of magnetic adatoms can also turn graphene from a trivial insulator to a $\mathrm{QAHE}{ }^{32182}$.

The above adatom adsorption studies suggest that in a realistic graphene sample, the topological state is the favored ground state. Therefore, this calculation provides evidence of the high possibility of realizing topological phases in graphene $e^{32}$.
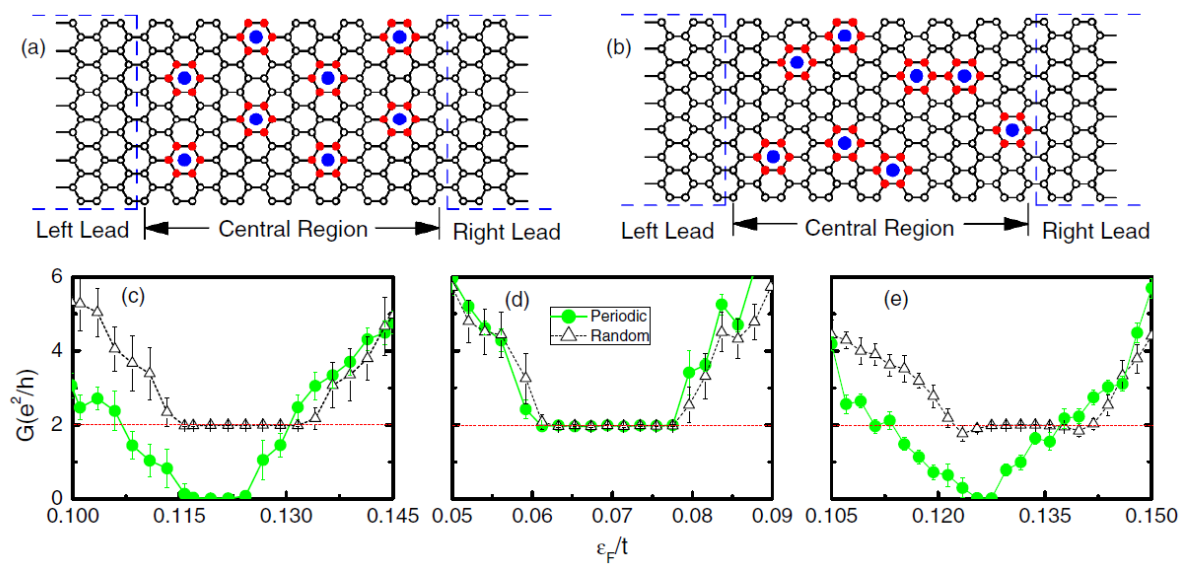

Fig. 7. (color online)(a)(b) Schematic of a two-terminal device with periodically and randomly distributed adatoms, respectively. Both adatom coverages are $11.1 \%$.(c) C(e) Comparison of conductances $\mathrm{G}$ between periodic and random adsorption as a function of Fermi level $\varepsilon_{F} \cdot(\mathrm{c}) \mathrm{C}(\mathrm{d})$ The site potential and intrinsic SOC are set to $U=0.36 t$ and $\lambda_{\mathrm{SO}}=0.016 t$. The adatom coverages are $11.1 \%$ in panel (c) and $6.25 \%$ in panel (d). In (e) the adatom coverage is $11.1 \%$. Circle and triangle symbols represent the periodic and random adatoms. The error bars denote the conductance fluctuations. Adapted from Ref $\underline{[0]}$

\section{METAL-INSULATOR TRANSITION}

Since it was first proposed by P. W. Anderson, the disorder induced metal-insulator transition has been a long lasting, interesting research issue in condensed matter physics 83 . According to scaling theory, the metal-insulator transition in a material system depends on its universality ensemble [symplectic, unitary and orthogonal] and dimension 84.86 . Initially, it was believed that the extended states (metal) could exist only in three-dimensional systems and that all the bulk states in two and one-dimensional systems were localized (insulator) 84 . Later, two exceptions were found in two-dimensional systems. One was a the system with strong spin-orbital coupling [symplectic ensemble]. The other was a system without time reversal symmetry [unitary ensemble]. The topological states always share these two features and then become the focus of research on the metal-insulator transition. In the past two decades, the metalinsulator transition in quantum Hall systems has been clearly understood 87 89. The extended state can only exist at the transition point between different plateaus. The critical exponents of the transition have also been obtained. In the last few years, there have been many studies of the metal-insulator transition in a QSHE 90 94. Their results can be summarized as follows (i for a QSHE with Rashba spin-orbital coupling, the system belongs to a sympletetic ensemble. A metallic phase emerges between the QSH phase and the normal insulator phase; (ii) for a QSHE without Rashba spin-orbital coupling, the system can be divided into two spin subsystems, both of which belong to unitary ensemble. A direct transition from a QSHE to a normal insulator is observed. Though the metal-insulator transition in two-dimensional topological states has been extensively studied, the transition properties in three-dimensional 
topological states are still not quite clear.

More recently, the Weyl semimetal (WSM), a gapless topological state in three-dimension, has been theoretically predicted and experimentally confirmed $18|19| 21$. Thus, it is timely to study the effects of disorder and metal-insulator transition both for their direct experimental relevance and for their fundamental value in advancing the understanding of the interplay between randomness and topological order. In reference ${ }^{33}$, we do so by using both numerical and analytical approaches. Different from the previous studies that only concentrate on single weyl node ${ }^{95}$, we focus on a more realistic tight-binding model with considering the interplay of opposite weyl nodes 98999 . By calculating the localization length $\frac{100}{1}$ and the Hall conductivity $101 \mid 102$, we obtain the complete phase of the system under all the disorder strength. Because the WSM has novel gapless excitations, i.e. the Weyl nodes in the bulk and Fermi arcs on the surface, we find an unexpected rich phase diagram, as shown in Fig. 8(c). With increasing disorder strength, the system undergoes multiple phase transitions. For example, one can obtain (i) 3D quantum anomalous Hall state (QAH) -3D diffusive anomalous Hall metal (metal) - normal insulator (NI); (ii) WSM - QAH-metal-NI ; (iii) WSMmetal-NI; (iv) NI-WSM-metal-NI in Fig. 8(a),(b),(d),(e) respectively. Through the comprehensive study of the phase diagram, we first address the important issue on the stability of the Weyl nodes and Fermi arcs against weak disorder. The weak disorder has important effects when the Weyl nodes are close in momentum space. The WSM to QAH and the NI to WSM transitions are caused by the pair annihilation near the zone boundary and pair nucleation at the zone center. Then, we find the the transition from WSM to metal by moderate disorder is unconventional. This transition takes place between two metallic states and is only enabled by the topological character of the Weyl nodes.
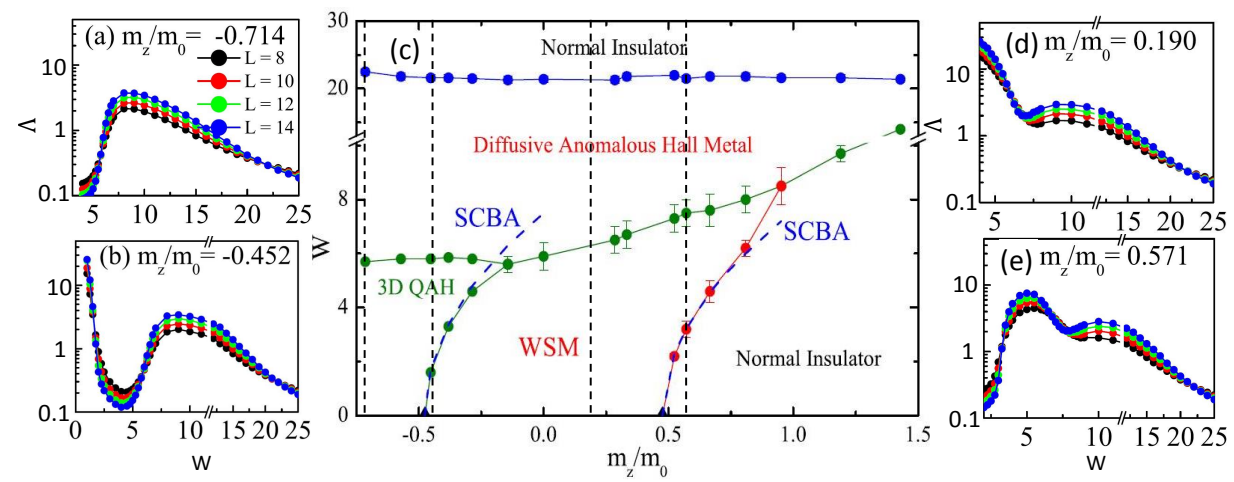

Fig. 8. (color online) (a),(b),(d),(e) Normalized localization length $\Lambda=\lambda(L) / L$ vs disorder strength $W$ for different masses $m_{z}$ [lines in subplot (c)]. $\lambda(L)$ is the localization length of a long bar sample with cross section $L \times L$. An increase in $\Lambda$ with $L$ signals a metal phase, while a decrease with $L$ signals an insulator phase. When $\Lambda$ is independent of $L$, this signals a critical point of the phase transition. (c) Phase diagram in the $W-m_{z}$ plane. The symbols guided by the solid lines were obtained from the normalized localization length. The dashed blue lines are the phase boundaries determined using the SCBA. In a finite layer sample, the 3D QAH, WSM and Diffusive anomalous Hall metal phases are distinguished by its Hall conductance being quantized and equivalent to to layer number, being quantized, and being non-quantized, respectively. Adapted from 33

It is worth noting that independent of our work, Liu et al also studied the disorder induced metal-insulator transition in 3D QAH layer systems and observed a rich phase diagram 103 . Moreover, the unconventional WSM to metal transition has also generate broad interest, and the critical exponent of the transition has been obtained $103+105$. 


\section{CONCLUSIONS AND OUTLOOK}

In this paper, based on reviewing our own disorder studies and other relevant works produced over the last few years, recent developments into the effects of disorder in topological states are briefly summarized. We show that all of weak, moderate and strong disorder can lead to exotic phenomena in various type of topological states. How these phenomena originate from the topologically nontrivial nature of topological states is also demonstrated. In spite of significant recent progress, there is nevertheless still plenty of room for further research into the effects of disorder in topological states. Before concluding the review, we discuss the opportunities for disorder studies in the future.

Experimental and material realization of disorder related topological phases. Seven years after its first prediction 30153 , the first signs of the experimental realization of the TAI phase have now been found. However, the host system is evanescently coupled waveguides 55106 . Due to the recent great advance in controlling the disorder strength ${ }^{107}$ and in the measurement of the local current ${ }^{108}$ in topological material, we expect the TAI phase can soon be confirmed in condensed matter systems. It is also to be noted that the topological phase in graphene has promising applications in information processing. We have demonstrated that both QSHE and QAHE states in graphene can easily be engineered through randomly adsorbing nonmagnetic/magnetic adatoms ${ }^{32}$. We also expect the experimental observation of these topological states.

Understanding the fundamental phenomena caused by the disorder. By now, our understanding of many disorder related phenomena is still limited. Firstly, it is a common belief that 2D unitary system is scaled to insulator except at some isolated critical points ${ }^{86}$. However, in reference ${ }^{109}$ and reference ${ }^{110}$, though the considered models are totally different, we find in both a novel metallic phase region that may emerge between QSHE and normal insulator phases. The physical origins for the metallic phase and the relationship between these two models should also be carefully addressed. Secondly, analogue to disorder induced Anderson localization benefits the observation of quantized Hall plateaus in QHE, the disorder also plays an important role in the experimental observation of the QSHE and QAHE ${ }^{44 / 45}$. Du et al find that dilute silicon impurity doping in InAs/GaSb quantum wells can greatly suppress residual bulk conductance and produce a perfect QSHE ${ }^{45}$. From the point of view of theory, despite the attempt to study the phenomena ${ }^{57}$, the mechanism for the effects of impurity doping on the transport properties of the system is still not clear. Significantly, there exist great puzzles regarding disorder effects in thin films of chromium-doped $(\mathrm{Bi}, \mathrm{Sb})_{2} \mathrm{Te}_{3}$, which is key to understand why the QAHE can only be observed at extremely low temperatures 111111 . Thirdly, type-II Weyl semimetals, which harbor unconventional Weyl nodes, have been proposed recently ${ }^{112}$. It would be interesting to study the stability of these Weyl nodes under strong disorder. Finally, dephasing effects also exist in realistic samples. A natural topic is how the transport properties of topological states are affected when both disorder and dephasing effects are considered 113 .

Acknowledgments: We are grateful to X. C. Xie, Q. Niu, Z. Q. Wang, Q.-F. Sun, J. R. Shi H. W. Liu, Z. H. Qiao, J. Feng, L. Wang, Z. B. Wang, D. W. Xu and C. Z. Chen for collaboration and for their important contributions reviewed in this paper. H. J. also appreciates the discussions with J. Liu, J. H. Gao, Y. G. Yao and S. Q. Shen. This Project supported by the National Natural Science Foundation of China (Grant No.11374219, No. 11474085 and No. 
* jianghuaphy@suda.edu.cn

1 Prange R E and Girvin S M 1987 The Quantum Hall Effect, New York, Springer

2 Hasan M Z, and Kane C L, 2010 Rev. Mod. Phys 823045

3 Qi X L and Zhang S C, 2011 Rev. Mod. Phys 831057

4 Shen S Q 2012 Topological Insulator, Springer

5 Weng H M, Yu R, Hu X, Dai X, and Fang Z, 2015 Adv. Phys. 64227.

${ }^{6}$ Ren Y F, Qiao Z H, Niu Q, 2015 arXiv: $\mathbf{1 5 0 9 . 0 9 0 1 6}$

7 Chiu C K, Teo J C Y, Schnyder A P, and Ryu S, 2016 arxiv: $\mathbf{1 5 0 5 . 0 3 5 3 5}$

8 Weng H M, Dai X, and Fang Z 2016 arxiv: 1603.04744

${ }^{9}$ Klizing K v, Dorda G, and Pepper M, 1980 Phys. Rev. Lett., 45494

10 Haldane F D M, 1988 Phys. Rev. Lett. 612015

11 Chang C Z, Zhang J S, Feng X, et al., 2013 Science 340167

12 Kane C L and Mele E J, 2005 Phys. Rev. Lett 95226801

13 Kane C L and Mele E J, 2005 Phys. Rev. Lett 95146802

${ }^{14} \mathrm{Fu}$ L, Kane C L, and Mele E J 2007 Phys. Rev. Lett 98106803

15 Moore J E and Balents L 2007 Phys. Rev. B 75 121306(R)

${ }^{16} \mathrm{Fu} \mathrm{L}, 2011$ Phys. Rev. Lett 106106802

17 Volovik G E 2002 JETP. Lett 7555

18 Wan X, Turner A M, Vishwanath A V and Savrasov S Y, 2011 Phys. Rev. B, 83205101

19 Lv B Q et al., 2015 Phys. Rev. $x 5$ 031013; Xu S Y et al., 2015 Science 349613

${ }^{20}$ Liu Z K, et al., 2014 Science 343, 864

21 Weng H, Fang C, Fang Z, Bernevig B A, and X Dai, 2015 Phys. Rev. X, 5011029

${ }^{22}$ Wang Z, Sun Y, Chen X Q, Franchini C, Xu G, Weng H, Dai X,and Fang Z, 2012 Phys. Rev. B 85, 195320; Wang Z, Weng H, Wu Q, Dai X, and Fang Z, 2013 Phys. Rev. B 88125427.

23 Thouless D J, Kohomoto M, Nightingale M P, and Nijs M D, 1982 Phys. Rev. Lett 49405

${ }^{24}$ König M, Wiedmann S, Bruene C, Roth A, Buhmann H, Molenkamp L W, Qi X L and Zhang S C, 2007 Science 318766

25 Zhang T, Cheng P, et al., 2009 Phys. Rev. Lett. 103266803

${ }^{26}$ Chen J et al., 2010 Phys. Rev. Lett, 105, 176602

27 Lu H Z, Shi J R, and Shen S Q, 2011 Phys. Rev. Lett. 107076801

28 Aji V 2012 Phys. Rev. B 85 241101(R)

29 Xiong J, et al., 2015 Science 350413

30 Jiang H, Wang L, Sun Q F and Xie X C, 2009 Phys. Rev. B 80165316

31 Jiang H, Liu H W, Feng J, Sun Q F, and Xie X C, 2014 Phys. Rev. Lett 112176601

32 Jiang H, Qiao Z H, Liu H W, Shi J R and Qian N, 2012 Phys. Rev. Lett 109116803

33 Chen C Z, Song J T, Jiang H, Sun Q F, Wang Z Q, and Xie X C, 2015 Phys. Rev. Lett 115246603

$34 \mathrm{Xu}$ C K and Moore J E, 2006 Phys. Rev. B, 73045322

35 For a brief review, see S. Datta 2006 chapter 4 of Electron Transport in Mesoscopic Systems, Cambridge University Press, Cambridge

36 Qiao Z H, Wang J, Sun Q F, and Guo H, 2009 Phys. Rev. B, 79205308

37 Sheng L, 2005 Phys. Rev. Lett 95136602

38 Pan H, Li X, Jiang H, Yao Y G, and Yang S Y, 2015 Phys. Rev B 91045404

${ }^{39}$ Li X, Liu H W, Jiang H, Wang F, and Feng J 2012 Phys. Rev. B, 90165412

${ }^{40}$ Li D F and Shi J R 2009 Phys. Rev. B 79241303

41 Zhang Y Y, Shen M, An X T, Sun Q F, Xie X C, Chang K, and Li S S, 2014 Phys. Rev. B, 90, 054205

42 Bernevig B A, Hughes T L and Zhang S C, 2006 Science 3141757

43 Qi X L, Wu Y S, and Zhang S C, 2006 Phys. Rev. B 74085308

${ }^{44}$ Knez I, Du R -R, and Sullivan G, 2011 Phys. Rev. Lett 107136603

45 Du L J, Knez I, Sullivan G, and Du R -R 2015 Phys. Rev. Lett 114096802

46 Kane C L, 2008 Nature Phys 4348

47 Guo H M, Lin Y and Shen S Q, 2014 Phys. Rev. B 115246603

48 Fukui T, and Hatsugai Y, 2015 J. Phys. Soc. Jpn, 84043703

49 Leeuw B d, Küpersbusch C, Juričić, and Fritz L, 2015 Phys. Rev. B 91235430

50 Wang Z B, Song J T, Liu H W, Jiang H and Xie X C 2015 New J. Phys. 17113040

51 Zhu K, Wu L, Gong X X, Xiao S H, Li S Y, Jin X F, Yao M Y, Qian D, Wu M, Feng J, Niu Q, Juan F D, Lee D H, 2014 arxiv $1403.0066 \mathrm{v} 1$

${ }^{52}$ Yao M Y, Zhu F F, Miao L, Han C Q, Yang F, Guan D D, Gao C L, Liu C H, Qian D, Jia J F, 2016 Sci. Rep 621326

${ }^{53} \mathrm{Li}$ J, Chu R L, Jain J K, and Shen S Q, 2009 Phys. Rev. Lett 102136806

${ }^{54}$ Guo H M, Rosenberg G, Refael G, and Franz M, 2009 Phys. Rev. Lett 105, 216601. 
55 Titum P, Lindner N H, Rechtsman M C, and Refael G 2015 Phys. Rev. Lett 114056801

56 Xing Y X, Zhang L, and Wang J 2011 Phys. Rev. B, 84035110

57 Xu D H, Gao J H, Liu C X, Sun J H, Zhang F C, and Zhou Y, 2014 Phys. Rev. B, 89195104

${ }^{58} \mathrm{Hu} \mathrm{L} \mathrm{H}, \mathrm{Xu}, \mathrm{D} \mathrm{H}$, Zhang F C, Zhou Y, 2016 arxiv:, 1604.00821

59 Qin W, Xiao D, Chang K, Shen S Q, and Zhang Z Y, 2015 arxiv: $\mathbf{1 5 0 9 . 0 1 6 6 6}$

${ }^{60}$ Borchmann J, Farrell A, and Pereg-Barnea T, 2016 Phys. Rev. B 93125133

61 Ryu S and Nomura K, 2012 Phys. Rev. B 85155138

62 Song J T, Prodan E, 2014 Phys. Rev. B 89224203

63 Song J T, Fine C, and Prodan E 2014 Phys. Rev. B 90184201

64 Orth C P, Sekera T, Bruder C, Schmidt T L 2016 Sci. Rep 624007

65 Su Y, Avishai Y, Wang X R 2016 arxiv: 1601.02541

${ }^{66}$ Groth C W, Wimmer M, Akhmerov A R, Tworzydlo J and Beenakker C W J, 2009 Phys. Rev. Lett 103196805

67 Bruus H, Flensberg K, 2004 Many-body Qumantum Theory in Condensed Matter Physics, Oxford University Press

68 Song J T, Liu H W, Jiang H, Sun Q F, and Xie X C, 2012 Phys. Rev. B 85195125

69 Prodan E, 2011 Phys. Rev. B 83, 195119

70 Xu D W, Qi J J, Liu J, Sacksteder V IV, Xie X C and Jiang H 2012 Phys. Rev. B 85195140

71 Zhang Y Y, Chu R L, Zhang F C, and Shen S Q 2012 Phys. Rev. B 85035107

72 Zhang Y Y, and Shen S Q 2012 Phys. Rev. B 88195145

73 Girschik A, Libisch F, and Rotter S. 2013 Phys. Rev. B 88, 014201

${ }^{74}$ Girschik A, Libisch F, and Rotter S. 2015 Phys. Rev. B 91, 214204

75 Chen L, Liu Q, Lin X, Zhang X and Jiang X 2012 New J. Phys. 14043028

76 Weeks C, Hu J, Alicea J, Franz M, and Wu R, 2010 Phys. Rev. X 1021001

$77 \mathrm{Hu}$ J, Alicea J, Wu R, and Franz M 2012 Phys. Rev. Lett 109266801

${ }^{78}$ Li Y C, Tang P Z, Chen P C, Wu J, Gu B L, Fang Y, Zhang S B, and Duan W H 2013 Phys. Rev. B 87245127

79 Qiao Z H, Yang S A, Feng W, Tse W K, Ding J, Yao Y, Wang J and Niu Q, 2010 Phys. Rev. B 82 161414(R)

80 Ding J, Qiao Z, Feng W, Yao Y and Niu Q 2011 Phys. Rev. B 84195444

81 Zhang H, Lazo C, Blügel S, Heinze S, and Mokrousov Y, 2012 Phys. Rev. Lett. 108056802

82 Qiao Z H, Jiang H, Li X, Yao Y G, and Niu Q, 2012 Phys. Rev. B 85115439

83 Anderson P W, 1958 Phys. Rev. 109, 1492

84 Abrahams E, Anderson P W, Licciardello D C, Ramkrishnan T V, 1979 Phys. Rev. Lett. 42673

85 Beenakker C W J, 1997 Rev. Mod. Phys 69731

86 Evers F and Mirlin A D 2008 Rev. Mod. Phys. 801355

87 Huckestein B, 1995 Rev. Mod. Phys 67357

88 Chalker J T, and Coddington P D 1988 J. Phys. C 212665

89 Slevin K and Ohtsuki T, 1997 Phys. Rev. Lett. 784083

90 Onoda M, and Nagaosa N, 2003 Phys. Rev. Lett 90206601

91 Onoda M, Avishai Y, and Nagaosa N, 2007 Phys. Rev. Lett 98076802

92 Yamakage A, Nomura K and Imura K I, Kuramoto Y. 2011 J. Phys. Soc. Jpn 80, 053703

93 Yamakage A, Nomura K and Imura K I, Kuramoto Y. 2013 Phys. Rev. B 87, 205141

94 Obuse H, Furusaki A, Ryu S, and Mudry C, 2007 Phys. Rev. B, 76075301

95 Sbierski B, Pohl G, Bergholtz E J, and Brouwer P W, 2014 Phys. Rev. Lett 113026602

96 Sbierski B, Bergholtz E J, and Brouwer P W, 2015 Phys. Rev. B 92115145

97 Pixley J H, Goswami P, and Sarma S D, 2015 Phys. Rev. Lett. 115076601

98 Nielsen H B, and Ninomiya, 1981 Nucl. Phys. B, 18520

99 Yang K Y, Lu Y M, and Ran Y, 2011 Phys. Rev. B 84075129

100 MacKinnon A and Kramer B, 1981 Phys. Rev. Lett 471546

101 Song J T, Prodan E, 2014 Euro. Phys. Lett. 10537001

102 Prodan E, 2011, J. Phys. A, 44113001

103 Liu S, Ohtsuki T, and Shindou R 2016 Phys. Rev. Lett 116066401

104 Shapourian H, and Hughes T L 2016 Phys. Rev. B 93075108

105 Bera S, Sau J D, and Roy B 2015 arxiv: 1507.07551v1

106 Stü tzer S, Rechtsman M C, Titum P, Plotnik Y, Lumer Y, Zeuner J M, Nolte S, Refael G, Lindner N, Segev M, and Szameit A 2015 Paper presented at CLEO: QELS Fundamental Science 10.1364CLEO ${ }_{Q}$ ELS.2015.FTh3D.2.

107 Liao J, Ou Y B, Feng X, Yang S, Lin C J, Yang W M, Wu K H, He K, Ma X C, Xue Q K, and Li Y Q, 2015 Phys. Rev. Lett 114216601

108 Nowack K C, Spanton E M, et al., 2013 Nature Mater 12787

109 Chen C Z, Liu H W, Jiang H, Sun Q F, Wang Z Q, and Xie X C, 2015 Phys. Rev. B 91214202

110 Qiao Z H, Wang K, Zhang L, Han Y L, Deng X Z, Jiang H, Yang S A, Wang J, Niu Q 2016 arxiv 1601.07367

111 Yu R, Zhang W, Zhang H J, Zhang S C, Dai X, and Fang Z, 2010 Science 32961

112 Soluyanov A A, Gresch D, Wang Z J, Wu Q S, Troyer M, Dai X, and Bernevig B A, 2015 Nature, 2015527

113 Liu H W, Jiang H, Sun Q F, and Xie X C, 2014 Phys. Rev. Lett 113046805 